\title{
Method Based on Sparse Signal Decomposition for Harmonic and Inter-harmonic Analysis of Power System
}

\author{
Lei Chen ${ }^{\dagger}$, Dezhong Zheng*, Shuang Chen** and Baoru Han $* * *$
}

\begin{abstract}
Harmonic/inter-harmonic detection and analysis is an important issue in power system signal processing. This paper proposes a fast algorithm based on matching pursuit (MP) sparse signal decomposition, which can be employed to extract the harmonic or inter-harmonic components of a distorted electric voltage/current signal. In the MP iterations, the method extracts harmonic/interharmonic components in order according to the spectrum peak. The Fast Fourier Transform (FFT) and nonlinear optimization techniques are used in the decomposition to realize fast and accurate estimation of the parameters. First, the frequency estimation value corresponding to the maxim spectrum peak in the present residual is obtained, and the phase corresponding to this frequency is searched in discrete sinusoids dictionary. Then the frequency and phase estimations are taken as initial values of the unknown parameters for Nelder-Mead to acquire the optimized parameters. Finally, the duration time of the disturbance is determined by comparing the inner products, and the amplitude is achieved according to the matching expression of the harmonic or inter-harmonic. Simulations and actual signal tests are performed to illustrate the effectiveness and feasibility of the proposed method.
\end{abstract}

Keywords: Matching pursuit algorithm, Harmonic, Inter-harmonic, Sinusoids dictionary, Fast Fourier Transform (FFT), Sparse decomposition, Optimal computation

\section{Introduction}

The increasing applications of nonlinear loads such as motor, transformer, arc furnace and domestic appliances, along with power electronic equipment in the power system result in a large amount of harmonic and interharmonic distortions. The presence of these distortions can cause adverse problems or effects such as voltage fluctuation, noise and vibration of motor, the decrease of power transmission efficiency, sub-synchronous oscillation of generator unit, malfunction of relay protection equipment [1-2]. Therefore, the analysis of harmonic and interharmonic in the electric signal is very important before taking actions to eliminate or suppress the problems mentioned above.

Different techniques have been developed in recent years for harmonic analysis, including Fast Fourier Transform (FFT) [3], short-time Fourier transform (STFT) [4], wavelet transform (WT) [5], Kalman filters [6], Prony method [7], Hilbert-Huang transform (HHT) [8], estimation of signal parameters via rotational invariance technique (ESPRIT) [9], independent component analysis (ICA) [10] and multiple signal classification (MUSIC) [11], etc.

The methods proposed above mostly assume that only

$\dagger$ Corresponding Author: Dept. of Electrical and information Engineering, Northeast Petroleum University, Qinhuangdao, China. (addisonqhd@163.com)

* School of Electrical Engineering, Yanshan University, China.

** School of Math and Information Technology, Hebei Normal University of Science and Technology, Qinhuangdao, China

*** Hainan Software Profession Institute, Hainan, China.

Received: July 8, 2016; Accepted: December 24, 2016 harmonics are present and the periodicity intervals are fixed. The literatures about the detection of transient harmonics or inter-harmonics are relatively small, and the discussion is incomplete. [12-13] analyzed the case that containing 5th transient harmonic only, and didn't provide phase detection results. The method in [14] can calculate the complexity of power harmonics and measure the frequency and power of transient harmonics, but didn't give the quantitative results of the parameters, and the selection rules of parameter need further study. In [15], a method for measurement of transient harmonics and inter-harmonics was proposed, but the simulation signal was quite different from real power signal. The method in [16] can detect the amplitude, frequency and duration time of the transient harmonics, but can't analyze phase information.

With the increasing diversity and complexity of the loads and power supply mode in power system, the existing methods need to be improved, and at the same time new detection techniques need to be explored also. In recent years, the sparse signal decomposition method based on overcomplete dictionary was applied to power system signal processing [17-19]. Gabor time-frequency atom method was used to analyze time-varying inter-harmonic signals, but the computation speed is not acceptable for practice [18]. The matching pursuit method based on Particle Swarm Optimization (PSO) was used to realize fast analysis of unsteady inter-harmonics, but the algorithm is limited to synchronous sampling [19].

This paper presents a method for extracting harmonics and inter-harmonics from power system signal using sparse 
signal decomposition technique with redundant dictionary. The proposed method extracts the parameters of harmonic and inter-harmonic components according to the spectrum energy by using MP iterative decomposition with overcomplete sinusoids dictionary. FFT, unconstrained nonlinear programming algorithm and the recursion based inner product computation are used to improve the speed and accuracy of the algorithm. To demonstrate the effectiveness of the technique, the proposed method is tested with different types of signals. The results show that the method is insensitive to spectral leakage, and is also proven to be capable of achieving better accuracy under noisy conditions comparing with other methods.

\section{MP Decomposition Based on Redundant Dictionary}

\subsection{Sparse representation based on redundant dictionary}

In recent years, sparse representation has been successfully used in many signal processing applications, including denoising, compression and feature extraction of various types of signals such as electrocardiosignal, radar signal and image signal, etc.

Redundant dictionary is constructed by function waveforms with a wide range of time-frequency distribution, such waveforms in the dictionary are called time-frequency atoms. The dictionary should contain a very large number of distinct structures for any phenomena or be constructed based on the prior information about time-frequency characteristics of the signals of interest, so that we could potentially identify or discriminate different phenomena that composing a signal. In this case, the dictionary is called redundant or over-complete, which can provide better representation of a composite signal with both stationary and non-stationary components by using hybrid elementary waveforms from different analytical functions.

For a predefined dictionary, an $N \times 1$ signal $f$ can be expressed as:

$$
\begin{gathered}
f=\psi \alpha=\sum_{n=0}^{S-1} \alpha_{n} \psi_{n} \\
\psi=\left[\psi_{0}, \psi_{1}, \psi_{2}, \ldots, \psi_{S-1}\right], \quad \alpha=\left[\alpha_{0}, \alpha_{1}, \alpha_{2}, \ldots, \alpha_{S-1}\right]^{\mathrm{T}}
\end{gathered}
$$

where $\psi_{n}$ are the time-frequency atoms in an $N \times S$ dictionary matrix $\psi$, and $\alpha_{n}$ are the sparse transformed coefficients in $S \times 1$ column vector $\alpha$. Unlike other timefrequency analysis techniques using a single or fixed basis matrix to express any signal, such as Fourier and wavelet, the sparse representation based on redundant dictionary can select adaptively the elements of the dictionary depending upon the local properties of signal $f$.

The sparse decomposition based on redundant dictionary can realize flexible decomposition of signals whose localizations in time and frequency vary widely, and the goal of which is to represent signal by least atoms with small enough error. Since the atoms selected have the most matching structure with the signal, the method can be used to realize features extraction and parameters detection of electrical signal. It is clear that the construction of suitable dictionary and the way to find out appropriate atoms are the two key problems to be solved.

\subsection{MP decomposition}

For the input signal $f$ expressed as (1), the sparse coefficients vector $\alpha$ can be estimated by solving the following optimization problem:

$$
\min _{\alpha}\|\alpha\|_{0} \text { subject to } f=\psi \alpha
$$

The above optimization problem can be solved using matching pursuit (MP), a nonlinear iterative greedy algorithms proposed by Mallat and Zhang [20].

Defining a dictionary $D=\left\{g_{\gamma}\right\}_{\gamma \in \Gamma}, g$ denotes the atoms in the dictionary, and $\Gamma$ is the set of all possible parameters $\gamma$ that define $g$, and $\left\|g_{\gamma}\right\|=1$. MP decomposes $f$ by projecting it on an atom of $D$ that matches $f$ almost at best. Let $g_{\gamma 0} \in D$, then $f$ can be decomposed into

$$
f=\left\langle f, g_{\gamma 0}\right\rangle g_{\gamma 0}+R
$$

where $R$ is the residual after approximating $f$ in the direction of $g_{\gamma 0}$. Since $g_{\gamma 0}$ is orthogonal to $R$, hence

$$
\|f\|^{2}=\left|<f, g_{\gamma 0}>\right|^{2}+\|R\|^{2}
$$

To minimize $\|R\|, g_{\gamma 0}$ should be selected to maximize $\left|<f, g_{\gamma 0}>\right|$.

MP algorithm then decomposes the residue $R$ following the above process, and this procedure is repeated each time on the following obtained residue. Thus $f$ can be approximated by the sum of orthogonal projections of the atoms in $D$. The decomposition process can be described as follows:

Let $R^{n}$ be the $n$th order residue $(n \geq 0)$, and $R^{0}=f$. An atom $g_{\gamma n} \in D$ that closely matches the residue $R^{n}$ is selected in each step, and satisfies

$$
g_{\gamma n}=\underset{\gamma \in \Gamma}{\arg \max }\left|\left\langle R^{n}, g_{\gamma}\right\rangle\right|
$$

The residue $R^{n}$ can be decomposed into

$$
R^{n}=\left\langle R^{n}, g_{\gamma n}\right\rangle g_{\gamma n}+R^{n+1}
$$

Carrying out this decomposition process iteratively up to order $M$, and the signal $f$ can be expressed as 


$$
f=\sum_{n=0}^{M-1}\left\langle R^{n}, g_{\gamma n}\right\rangle g_{\gamma_{n}}+R^{M}
$$

where $\sum^{M-1}\left\langle R^{n}, g_{\gamma n}\right\rangle g_{\gamma n}$ is the $M$-term approximation of $f$, $R^{M}$ is the $M$ th order residue. The iteration stops when the specified residual threshold or a fixed number of steps is achieved.

As we can see, MP algorithm obtains $M$ atoms up to step $M$, and the signal $f$ can be represented as the linear combination of these atoms, the decomposition coefficients $\alpha_{n}=\left\langle R^{n}, g_{\gamma n}\right\rangle, n=0,1,2, \ldots, M-1$.

\subsection{Sinusoids dictionary}

The time-frequency characteristic of atoms in the dictionary that used to decompose the signal $f$ should adapt to the local structure of $f$. The power system harmonics and inter-harmonics are a series of sinusoidal waveforms, so sinusoids dictionary is used in this paper, the complex expression of the sinusoids atom is:

$$
g_{\gamma}(t)=K e^{j \omega t}
$$

and the real sinusoids atom can be expressed as:

$$
g_{\gamma}(t)=K \cos (\omega t+\varphi)
$$

where $K$ is the normalized amplitude of the atom, $\omega$ denotes the frequency, $\varphi$ denotes the phase, and $\gamma=(\omega, \varphi)$ is the index of the parameters set.

The dictionary $D$ may have an infinite number of elements if the parameters of the atom are successive, in order to implement MP efficiently, the parameters of the atom should be discretized, that is to construct a discretized sub-dictionary $D_{\alpha}=\left(g_{\gamma}\right)_{\gamma \in \Gamma \alpha} \in D, \Gamma_{\alpha}$ is the discrete subset of $\Gamma$, and then we translate (5) into:

$$
g_{\gamma n}=\underset{\gamma \in \Gamma_{\alpha}}{\arg \max }\left|\left\langle R^{n}, g_{\gamma}\right\rangle\right|
$$

Referring to the discretization method of Gabor atom suggested in [20] to construct a sub-dictionary $D_{\alpha}$, and the atoms of sinusoids dictionary can be discretized as:

$$
\gamma=(\omega, \varphi)=\left(k 2^{-j} \Delta \omega, i \Delta \varphi\right)
$$

where $\Delta \varphi=\pi / 6, \Delta \omega=\pi, 0 \leq k<2^{j+1}, 0 \leq i \leq 12$, $0<j \leq \log _{2} N$, and $N$ is the sampling number.

\section{Harmonic/inter-harmonic Detection Based on FMP}

The number of atoms is still very large in the discretized sinusoids dictionary, which will leads to high computation complexity of MP. And also much error will be introduced inevitably for the discontinuity of the parameters. Here, a Fast Matching Pursuit (FMP) method is proposed to realize rapid calculation and achieve more precise matching of parameters.

\subsection{Preliminary estimation of matching parameters}

Let $f_{\mathrm{s}}$ denote the sampling frequency, and $N$ be the signal length. Firstly, FFT is used to find the initial guess frequency of the component, the spectrum $\lambda$ of $R^{n}$ with the maximum amplitude is obtained by FFT in each iteration, and the corresponding frequency $\omega_{\lambda}$ is computed as:

$$
\omega_{\lambda}=2 \pi(\lambda-1) f_{s} / N \quad(\mathrm{rad} / \mathrm{s})
$$

After obtaining $\omega_{\lambda}$, the phase $\varphi_{\lambda}$ corresponding to $\omega_{\lambda}$ that satisfies (10) will be searched by traversing the discretized dictionary in terms of $i \Delta \varphi(\Delta \varphi=\pi / 6,0 \leq i \leq 12)$. Here we only get the estimate values of frequency and phase, subsequently, optimization calculation method will be used to correct or adjust the value of $\omega_{\lambda}$ and $\varphi_{\lambda}$, so as to decrease or eliminate the errors induced by spectrum leakage and discontinuity of parameters.

\subsection{Parameters optimization}

In every iteration, searching the atoms satisfying (10) in the sub-dictionary $D_{\alpha}$ can be dealt with as a nonlinear programming problem, defining the objective function as:

$$
F(\omega, \varphi)=-\left|<R^{n}, g_{\gamma}>\right|
$$

Nelder-Mead algorithm is a nonlinear optimal method based on simplex, which uses direct search strategy without calculating derivative of the objective function and also have nothing to do with Hessian matrix operation. The method is especially suitable for solving the extreme value problem with not many variables.

Taking the estimation values of $\omega_{\lambda}$ and $\varphi_{\lambda}$ as the initial solution of the Nelder-Mead algorithm and solving the minimum problem without constrains as:

$$
\min F(\omega, \varphi)
$$

Firstly, for the 2 variables $\omega$ and $\varphi$ of the objective function (13), constructing a simplex of 2-dimensional space that containing 3 points based on $\left(\omega_{\lambda}, \varphi_{\lambda}\right)$, and every point is a 2-dimensional vector, the 3 points are $\left(\omega_{\lambda}, \varphi_{\lambda}\right),\left(\omega_{\lambda 1}, \varphi_{\lambda}\right)$ and $\left(\omega_{\lambda}, \varphi_{\lambda 1}\right)$ respectively, where $\omega_{\lambda 1}=(1+\Delta) \omega_{\lambda}, \varphi_{\lambda 1}=(1+\Delta) \varphi_{\lambda}$ and $\Delta=0.005$. Then calculating and comparing the function values at these 3 points, and update the simplex by reflection, expansion, and contraction at each stage [21]. The action contracts the simplex towards the lowest point, and will eventually obtaining the minimum or the optimal point $\left(\omega_{\lambda n}, \varphi_{\lambda n}\right)$ as the solution of (14). $\left(\omega_{\lambda n}, \varphi_{\lambda n}\right)$ is the final optimal atomic 
parameters matching with the residual $R^{n}$ in MP, so that the real atom can be written as:

$$
g_{\gamma n}(t)=K \cos \left(\omega_{\lambda n} t+\varphi_{\lambda n}\right)
$$

\subsection{Searching for temporal supports}

The disturbance duration should be determined for the measurement of transient harmonic. We denote $t_{s}$ and $t_{e}$ as the start and end time of the disturbance respectively, and the corresponding sample sequences are $k_{s}$ and $k_{e}$, with $t_{s}=k_{s} / f_{s}$ and $t_{e}=k_{e} / f_{s}$. Thus, the atoms expression with temporal supports can be expressed as:

$$
g_{\gamma}(t)=K \cos (\omega t+\varphi)\left[u\left(t-t_{s}\right)-u\left(t-t_{e}\right)\right]
$$

with $K$ such that $\left\|g_{\gamma}(t)\right\|=1$, where $u(t)$ is the unit step function. The discrete version of (16) is:

$$
g_{\gamma}(k)=K \cos \left(\frac{\omega k}{f_{s}}+\varphi\right)\left[u\left(k-k_{s}\right)-u\left(k-k_{e}\right)\right]
$$

where $k=1,2, \ldots \ldots, N$, and $k_{s} \leq k_{e}$, and the vector form of (17)can be represented as:

$$
g_{\gamma}=\left[g_{\gamma}(1), g_{\gamma}(2), \ldots, g_{\gamma}\left(k_{s}\right), \ldots, g_{\gamma}(N)\right]^{\mathrm{T}}
$$

In order to determine the value of $k_{s}$ and $k_{e}$, we need to traverse all the possible values. $N$ multiplications of real numbers are needed if we compute the inner product $\left\langle R^{n}, g_{\gamma}\right\rangle$ directly for every group of $k_{s}$ and $k_{e}$, which is time consuming. We suppose that $\gamma_{a}=\left(\omega, \varphi, k_{s}, k_{e}\right)$ and $\gamma_{b}=$ $\left(\omega, \varphi, k_{s}, k_{e}-1\right)$, and the atom corresponding to $\gamma_{a}$ is:

$$
g_{\gamma_{a}}=\left[g_{\gamma_{a}}(1), g_{\gamma_{a}}(2), \ldots, g_{\gamma_{a}}\left(k_{e}\right), \ldots, g_{\gamma_{a}}(N)\right]^{\mathrm{T}}
$$

then the unit-norm atom corresponding to $\gamma_{b}$ can be given by:

$$
g_{\gamma_{b}}=\frac{g_{\gamma_{a}}-\left[0,0, \ldots, g_{\gamma_{a}}\left(k_{e}\right), \ldots, 0\right]^{\mathrm{T}}}{\sqrt{1-g_{\gamma_{a}}\left(k_{e}\right)^{2}}}
$$

and further we have:

$$
\begin{aligned}
\left\langle R^{n}, g_{\gamma_{b}}\right\rangle & =\left\langle R^{n}, \frac{g_{\gamma_{a}}-\left[0,0, \ldots, g_{\gamma_{a}}\left(k_{e}\right), \ldots, 0\right]^{\mathrm{T}}}{\sqrt{1-g_{\gamma_{a}}\left(k_{e}\right)^{2}}}\right\rangle \\
& =\frac{\left\langle R^{n}, g_{\gamma_{a}}\right\rangle-R^{n}\left(k_{e}\right) \cdot g_{\gamma_{a}}\left(k_{e}\right)}{\sqrt{1-g_{\gamma_{a}}\left(k_{e}\right)^{2}}}
\end{aligned}
$$

Formula (21) shows that if we have known $g_{\gamma_{a}}$ and $\left\langle R^{n}, g_{\gamma_{a}}\right\rangle$, then $\left\langle R^{n}, g_{\gamma_{b}}\right\rangle$ can be calculated recursively with very low computational complexity. Thus, we can recursively obtain all of the inner products corresponding to all values of $k_{s}$ and $k_{e}$ quickly. Finally, $\left(k_{s n}, k_{e n}\right)$ that gives the largest inner product is used as the best temporal support, and we obtain:

$$
g_{\gamma n}(t)=K \cos \left(\omega_{\lambda n} t+\varphi_{\lambda n}\right)\left[u\left(t-t_{s n}\right)-u\left(t-t_{e n}\right)\right]
$$

Hence the amplitude of the harmonic or inter-harmonic can be achieved by:

$$
A_{n}=\max \left(<R^{n}, g_{\gamma n}>g_{\gamma n}\right)
$$

The harmonic/inter-harmonic extracted at the $n$th iteration of MP can be written as:

$$
g_{\gamma n}(t)=A_{n} \cos \left(\omega_{\lambda n} t+\varphi_{\lambda n}\right)\left[u\left(t-t_{s n}\right)-u\left(t-t_{e n}\right)\right]
$$

\subsection{Disturbances extraction steps of FMP method}

The steps of FMP are as follows:

(a) The frequency estimation $\omega_{\lambda}$ corresponding to the maximum peak of the spectrum of $R^{n}$ is obtained using FFT.

(b) With the frequency $\omega_{\lambda}$ obtained in step (a), search $\varphi_{\lambda}$ that satisfying (10) in the discrete sub-dictionary $D_{\alpha}$ according to $i \Delta \varphi$.

(c) Take $\left(\omega_{\lambda}, \varphi_{\lambda}\right)$ obtained in (a) and (b) as the initial value, compute the optimized frequency $\omega_{\lambda n}$ and phase $\varphi_{\lambda n}$ using Nelder-Mead algorithm.

(d) Find $t_{s n}$ and $t_{e n}$ using the simplified inner product method based on recursion showed in (21).

(e) Compute the expression $\alpha_{n} g_{\gamma n}=<R^{n}, g_{\gamma n}>g_{\gamma n}$ that matching with the harmonic or inter-harmonic disturbance, and get the amplitude $A_{n}$ of the disturbance.

Stop the process when the norm of residual $R^{n}$ is lower than a specified threshold or the maximum number of iteration is reached, otherwise repeat (a) to (e) iteratively.

\section{Case Studies and Experimental Results}

This section presents the results of simulated and actual signal case studies carried out using MATLAB to evaluate the performance of the proposed scheme. The following cases are considered. To simplify the magnitude values, per unit $(\mathrm{pu})$ value is used.

\subsection{Comparison with other methods}

FFT is widely employed for harmonic analysis because of its faster computational capability. However, for the presence of inter-harmonic and the fluctuation of grid frequency, it is hard to realize strict synchronous sampling, and which leads to spectrum leakage and picket fence 
effect, so the direct application of FFT for harmonic analysis is inaccurate and infeasible in this situation. Researches show that the improved window and interpolation FFT algorithms can reduce the undesirable errors caused by the spectral leakage and picket fence effect, and realize high precision harmonic detection.

But, for the intrinsic performance limitations of FFT, FFT based algorithms can not be used to analyze nonstationary signals with time-frequency localization properties. For example, if there are transient harmonics or inter-harmonics, other methods should be introduced, because the existing FFT based methods cannot solve it with satisfaction. Now, a series of other methods have been proposed to analyze harmonic and inter-harmonic, here the performance of the proposed method is compared with some of the methods available in the recent literatures.

\subsubsection{Analysis of steady harmonic and inter-harmonic}

The same model and sampling parameter settings as [22] are used here and the sampling rate $f_{s}$ is $6400 \mathrm{~Hz}$, the signal length $N$ is 2048 . To analyze the behavior of the method in the presence of both harmonics and inter-harmonics, a voltage signal containing the fundamental component plus the fifth and seventh harmonic components, along with the inter-harmonic of frequency $170 \mathrm{~Hz}$ presented in [22] is used here, and the signal is mathematically represented by:

$$
\begin{aligned}
f(t)= & \cos (2 \pi \times 50 t)+0.2 \cos (2 \pi \times 170 t)+ \\
& 0.12 \cos (2 \pi \times 250 t)+0.08 \cos (2 \pi \times 350 t)
\end{aligned}
$$

Noise is unwanted electrical signals with broadband spectral content typically lower than $200 \mathrm{kHz}$ superimposed upon the power system voltage or current. The frequency range and magnitude level of noise depend on the source that produces the noise and the system characteristics. Typical magnitude of noise is less than $1 \%$ of the voltage or current magnitude, thus the corresponding signal-tonoise ratio (SNR) is more than $40 \mathrm{~dB}$. To study the effect of noise on the performance of the proposed method, Gaussian white noise is added to signal $f(t)$ shown in (25), and signal with lower SNR of $30 \mathrm{~dB}$ is used here.

In the same simulation conditions, the results of FMP method is compared with the results of 3 other methods including Root-MUSIC, Beamspace Root-MUSIC and TLS-ESPRIT mentioned in literature [22]. Table 1 and 2 gives the relative errors of frequency and amplitude testing results of the 4 different methods respectively.

Table 1. Comparison of relative errors of frequency (\%)

\begin{tabular}{c|c|c|c|c}
\hline Frequency & FMP & $\begin{array}{c}\text { Beamspace } \\
\text { Root-MUSIC }\end{array}$ & $\begin{array}{c}\text { Root- } \\
\text { MUSIC }\end{array}$ & $\begin{array}{c}\text { TLS- } \\
\text { ESPRIT }\end{array}$ \\
\hline $50 \mathrm{~Hz}$ & 0.0016 & 0.0140 & 0.0240 & 0.0500 \\
\hline $170 \mathrm{~Hz}$ & 0.0017 & 0.0141 & 0.0106 & 0.0229 \\
\hline $250 \mathrm{~Hz}$ & 0.0026 & 0.0084 & 0.0348 & 0.0584 \\
\hline $350 \mathrm{~Hz}$ & 0.0004 & 0.0074 & 0.0140 & 0.0657 \\
\hline
\end{tabular}

Table 2. Comparison of relative errors of amplitude (\%)

\begin{tabular}{c|c|c|c|c}
\hline Amplitude & FMP & $\begin{array}{c}\text { Beamspace } \\
\text { Root-MUSIC }\end{array}$ & $\begin{array}{c}\text { Root- } \\
\text { MUSIC }\end{array}$ & $\begin{array}{c}\text { TLS- } \\
\text { ESPRIT }\end{array}$ \\
\hline $1 \mathrm{pu}$ & 0.03 & 0.10 & 1.00 & 0.40 \\
\hline $0.2 \mathrm{pu}$ & 0.25 & 1.50 & 4.00 & 2.50 \\
\hline $0.12 \mathrm{pu}$ & 0.33 & 1.67 & 7.50 & 2.50 \\
\hline $0.08 \mathrm{pu}$ & 0.63 & 1.25 & 3.75 & 3.75 \\
\hline
\end{tabular}

It is found that the test results of the proposed FMP method are more close to the preset values compared with other methods, and the frequency and amplitude relative errors of FMP are one order of magnitude lower than the other three methods. In addition, the SNR of the reconstructed signal is more than $50 \mathrm{~dB}$ if we reconstruct the components extracted from the original signal, which is well above $30 \mathrm{~dB}$. Therefore, we may consider that the proposed method can detect the harmonic and interharmonic parameters accurately and isolate the noise efficiently.

\subsubsection{Analysis of transient harmonic}

In order to verify the detection performance of the proposed method for transient harmonic, the same sampling setting $\left(f_{\mathrm{s}}=3200 \mathrm{~Hz}, N=1280\right)$ and signal model that contains fundamental, transient harmonics of $3^{\text {rd }}$ and $7^{\text {th }}$ as literature [16] are used here. Table 3 shows a comparative estimation of parameters for $3^{\text {rd }}$ and $7^{\text {th }}$ harmonic components respectively using both FMP and LMD methods. From these figures, it is verified that proposed FMP method estimates more accurately as compared to LMD method.

Table 3. Comparison of parameter estimation results

\begin{tabular}{c|c|c|c|c}
\hline Harmonic & Parameters & Set values & LMD method & FMP method \\
\hline \multirow{4}{*}{$3^{\text {rd }}$} & $A(\mathrm{pu})$ & 0.5 & 0.4985 & 0.5000 \\
\cline { 2 - 5 } & $f(\mathrm{~Hz})$ & 150 & 150.1000 & 149.9991 \\
\cline { 2 - 5 } & $t_{\mathrm{s}}(\mathrm{s})$ & 0.12 & 0.1206 & 0.1197 \\
\cline { 2 - 5 } & $t_{\mathrm{e}}(\mathrm{s})$ & 0.24 & 0.2417 & 0.2400 \\
\hline \multirow{4}{*}{$7^{\text {th }}$} & $A(\mathrm{pu})$ & 0.3 & 0.2993 & 0.3000 \\
\cline { 2 - 5 } & $f(\mathrm{~Hz})$ & 350 & 349.7000 & 350.0000 \\
\cline { 2 - 5 } & $t_{\mathrm{s}}(\mathrm{s})$ & 0.12 & 0.1205 & 0.1197 \\
\cline { 2 - 5 } & $t_{\mathrm{e}}(\mathrm{s})$ & 0.24 & 0.2418 & 0.2400 \\
\hline
\end{tabular}

\subsection{Analysis with fundamental frequency variation}

For performance evaluation of the proposed method at off-nominal frequency, a variation of $\pm 0.5 \mathrm{~Hz}$ is considered here. A sampling frequency of $3200 \mathrm{~Hz}$ and a SNR of $40 \mathrm{~dB}$ are considered, simulation was performed on records with 2048 samples. The signal used for estimation, besides the fundamental frequency, contains common harmonics of the $2^{\text {nd }}, 3^{\text {rd }}, 4^{\text {th }}, 5^{\text {th }}$ and inter-harmonics of $239 \mathrm{~Hz}$ and $256.7 \mathrm{~Hz}$, and the amplitude of even harmonics and inter-harmonics are very close to the noise. This kind of signal is mathematically represented by: 


$$
\begin{aligned}
& f(t)=\cos \left(2 \pi f_{1} t+10^{\circ}\right)+0.02 \cos \left(2 \pi f_{2} t\right)+ \\
& 0.14 \cos \left(2 \pi f_{3} t+57^{\circ}\right)+0.035 \cos \left(2 \pi f_{4} t+83^{\circ}\right) \\
& +0.1 \cos \left(2 \pi f_{5} t\right)+0.04 \cos \left(2 \pi \times 239 t+105^{\circ}\right) \\
& +0.015 \cos \left(2 \pi \times 256.7 t+35^{\circ}\right)+\delta(t)
\end{aligned}
$$

where $\delta(t)$ is Gaussian white noise.

Table 4 shows the relative frequency estimation errors of all components of the signal at different power frequency variations. Table 5 and 6 show the amplitude and phase estimation errors of fundamental, 2nd harmonic and interharmonic of $256.7 \mathrm{~Hz}$ respectively. The estimation results of the 2nd harmonic and $256.7 \mathrm{~Hz}$ inter-harmonic are chosen because of their weak amplitude close to noise.

From Table 4, it can be seen that the relative frequency errors of fundamental, harmonics and inter-harmonics are all less than $0.02 \%$, and for most cases the errors are less than $0.005 \%$. Table 3 shows that the proposed method possesses good frequency detection accuracy with typical noise amplitude of $1 \%$ of the signal magnitude when the power frequency varies from $49.5 \mathrm{~Hz}$ to $50.5 \mathrm{~Hz}$.

Table 5 and 6 show that for fundamental, the relative estimation errors of amplitude is less than $0.1 \%$, and the absolute estimation errors of phase is less than $0.2^{\circ}$. Though the inter-harmonic frequency of $256.7 \mathrm{~Hz}$ is very close to the frequency of $5^{\text {th }}$ harmonic, and the amplitude of which is commensurate with noise, the proposed method detected the amplitude and the phase information with relatively small errors.

The fundamental frequency and its corresponding

Table 4. Relative estimation errors of frequency using FMP (\%)

\begin{tabular}{c|c|c|c|c|c|c}
\hline & $49.5 \mathrm{~Hz}$ & $49.7 \mathrm{~Hz}$ & $49.9 \mathrm{~Hz}$ & $50.1 \mathrm{~Hz}$ & $50.3 \mathrm{~Hz}$ & $50.5 \mathrm{~Hz}$ \\
\hline $1^{\text {st }}$ & 0.0010 & 0.0014 & 0.0020 & 0.0024 & 0.0020 & 0.0016 \\
\hline $2^{\text {nd }}$ & 0.0174 & 0.0006 & 0.0141 & 0.0056 & 0.0147 & 0.0034 \\
\hline $3^{\text {rd }}$ & 0.0041 & 0.0011 & 0.0027 & 0.0062 & 0.0019 & 0.0014 \\
\hline $4^{\text {th }}$ & 0.0012 & 0.0007 & 0.0038 & 0.0014 & 0.0041 & 0.0018 \\
\hline $5^{\text {th }}$ & 0.0031 & 0.0016 & 0.0084 & 0.0022 & 0.0005 & 0.0086 \\
\hline $239 \mathrm{~Hz}$ & 0.0003 & 0.0008 & 0.0003 & 0.0012 & 0.0001 & 0.0014 \\
\hline $256.7 \mathrm{~Hz}$ & 0.0075 & 0.0069 & 0.0086 & 0.0046 & 0.0119 & 0.0110 \\
\hline
\end{tabular}

Table 5. Relative estimation errors of amplitude for fundamental, $2^{\text {nd }}$ harmonic and inter-harmonic of 256.7 $\mathrm{Hz}$ at different fundamental frequency (\%)

\begin{tabular}{c|c|c|c|c|c|c}
\hline & $49.5 \mathrm{~Hz}$ & $49.7 \mathrm{~Hz}$ & $49.9 \mathrm{~Hz}$ & $50.1 \mathrm{~Hz}$ & $50.3 \mathrm{~Hz}$ & $50.5 \mathrm{~Hz}$ \\
\hline $1^{\text {st }}$ & 0.07 & 0.08 & 0.07 & 0.02 & 0.08 & 0.05 \\
\hline $2^{\text {nd }}$ & 0.5 & 2.0 & 1.0 & 1.5 & 0.5 & 1.0 \\
\hline $256.7 \mathrm{~Hz}$ & 1.33 & 1.33 & 1.33 & 2.00 & 2.67 & 4.00 \\
\hline
\end{tabular}

Table 6. Absolute estimation errors of phase for fundamental, $2^{\text {nd }}$ harmonic and inter-harmonic of $256.7 \mathrm{~Hz}$ at different fundamental frequency $\left({ }^{\circ}\right)$

\begin{tabular}{c|c|c|c|c|c|c}
\hline & $49.5 \mathrm{~Hz}$ & $49.7 \mathrm{~Hz}$ & $49.9 \mathrm{~Hz}$ & $50.1 \mathrm{~Hz}$ & $50.3 \mathrm{~Hz}$ & $50.5 \mathrm{~Hz}$ \\
\hline $1^{\text {st }}$ & 0.1426 & -0.0220 & 0.1591 & 0.0415 & -0.1910 & 0.0073 \\
\hline $2^{\text {nd }}$ & 1.5450 & -0.5853 & -2.2733 & -0.4636 & 1.3644 & 0.6940 \\
\hline $256.7 \mathrm{~Hz}$ & 0.6611 & 0.5961 & 2.4172 & 1.0356 & 3.8145 & 2.8373 \\
\hline
\end{tabular}

harmonic frequency in (26) are detected accurately by using FMP method, and the week inter-harmonic components close to the $5^{\text {th }}$ harmonic are distinguished also. The results demonstrate that the proposed method can effectively overcome the impact of the variation of the system frequency, and the relative errors of frequency have very small change with system frequency variation.

\subsection{Analysis with complicated harmonic and inter- harmonic}

For the complicated electric power signal that containing both steady and transient harmonic and inter-harmonic, the signal to be analyzed is modeled as:

$$
\begin{aligned}
& f(t)=\cos \left(2 \pi \times 50 t+60^{\circ}\right)+0.15 \cos (2 \pi \times 150 t) . \\
& {[u(t-0.12813)-u(t-0.32)]+0.12 \cos (2 \pi \times 250 t) .} \\
& {[u(t)-u(t-0.09375)]+0.1 \cos (2 \pi \times 350 t)+} \\
& 0.05 \cos \left(2 \pi \times 27 t+150^{\circ}\right)+0.015 \cos (2 \pi \times 326 t)+ \\
& 0.02 \cos \left(2 \pi \times 100 t+90^{\circ}\right)+0.08 \cos (2 \pi \times 550 t) . \\
& {[u(t-0.14375)-u(t-0.22594)]+0.017 \cos (2 \pi \times 170 t) .} \\
& {[u(t-0.0625)-u(t-0.32)]}
\end{aligned}
$$

The sampling frequency of $3200 \mathrm{~Hz}$ is used here, and $N=1024$. The waveforms extracted from $f(t)$ by using FMP are showed in Fig. 1. Table 7 shows the parameter estimation results of the extracted waveforms.

In Fig. $1, u_{1}$ is the fundamental component, and $u_{2} \sim u_{9}$ are the steady or transient harmonic/inter-harmonic disturbance waveforms extracted from $f(t)$. It can be found

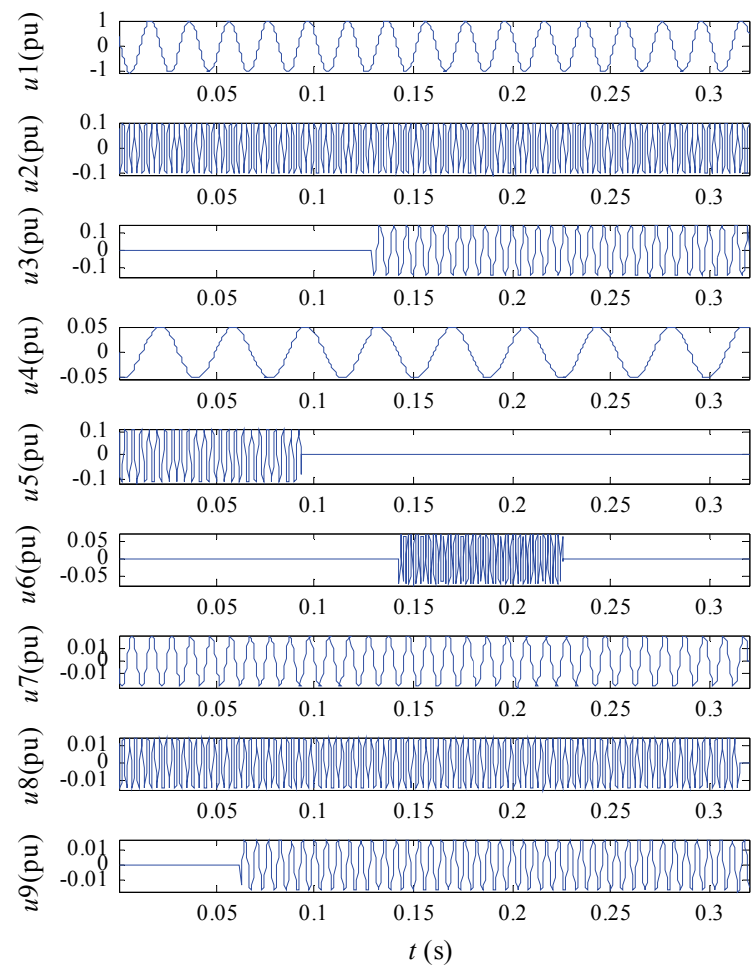

Fig. 1. Extracted waveforms from simulated signal $f(t)$ 


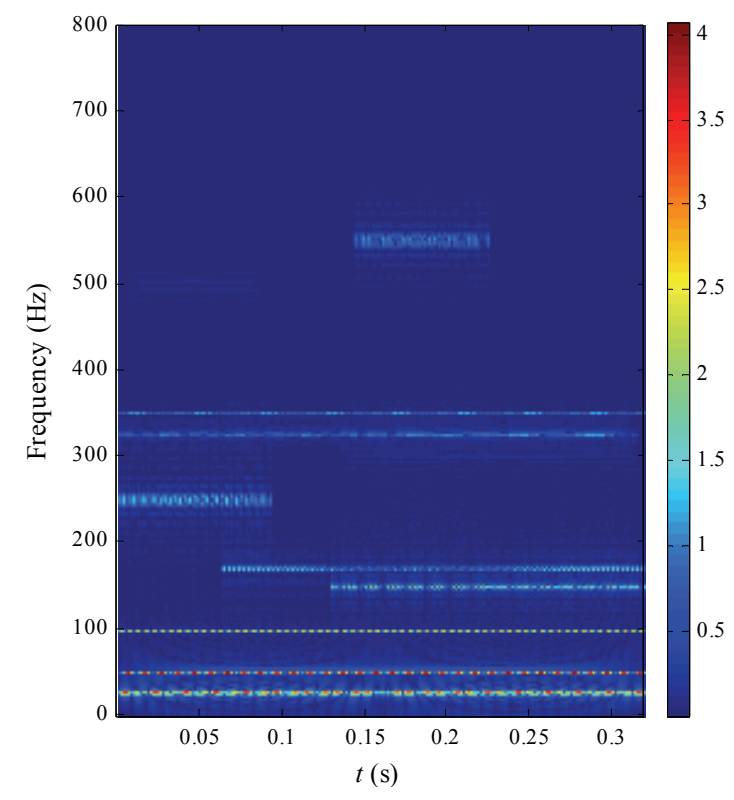

Fig. 2. Wigner-Viller distribution of the atoms corresponding to coherent components of the signal

Table 7. Parameter estimation results of the extracted waveforms

\begin{tabular}{c|c|c|c|c|c}
\hline & amplitude $(\mathrm{pu})$ & frequency $(\mathrm{Hz})$ & phase $\left(^{\circ}\right)$ & $t_{\mathrm{s}}(\mathrm{s})$ & $t_{\mathrm{e}}(\mathrm{s})$ \\
\hline$u_{1}$ & 0.9995 & 49.9980 & 60.1455 & 0.00031 & 0.32031 \\
\hline$u_{2}$ & 0.1007 & 349.9863 & -2.8017 & 0.00031 & 0.32031 \\
\hline$u_{3}$ & 0.1508 & 149.9522 & - & 0.12906 & 0.32000 \\
\hline$u_{4}$ & 0.0502 & 26.9959 & 150.3442 & 0.00063 & 0.32031 \\
\hline$u_{5}$ & 0.1196 & 250.0866 & 0.8882 & 0.00031 & 0.09375 \\
\hline$u_{6}$ & 0.0788 & 549.9785 & - & 0.14219 & 0.22625 \\
\hline$u_{7}$ & 0.0201 & 100.0201 & 95.1301 & 0.00031 & 0.31969 \\
\hline$u_{8}$ & 0.0147 & 326.0609 & 0.2229 & 0.00031 & 0.31531 \\
\hline$u_{9}$ & 0.0171 & 170.0642 & - & 0.06219 & 0.32031 \\
\hline
\end{tabular}

from expression (27) and Table 7 that the proposed method can estimate the amplitude, frequency, phase, start and end time of the disturbances with high precision from complicated multi-frequency electric power signal.

Fig. 2 shows the Wigner-Viller distribution of the atoms that best matching the signal structures extracted by FMP. It is clearly visible from Fig. 2 that there is no cross-term interference in the time-frequency distribution, thus avoiding the spectrum aliasing, and we can distinguish the information of frequency and duration time of each component clearly from the distribution diagram.

Furthermore, the following two cases are also considered. Case 1: adding white noise to the signal (27) and the SNR of $40 \mathrm{~dB}$ is considered. Case 2: the fundamental frequency in (27) varies from $49.5 \mathrm{~Hz}$ to $50.5 \mathrm{~Hz}$ are simulated. Similar extraction effectiveness as Table 7 can be obtained, so the results are not shown here for simplification.

\subsection{Results and analysis of actual power system data}

In this section, the decompositions of actual voltage
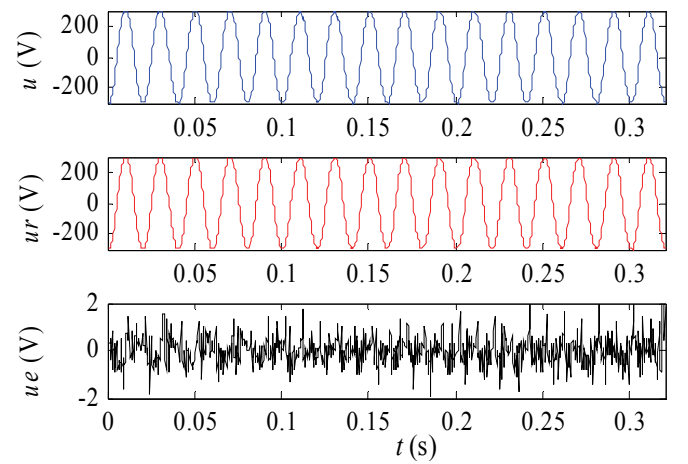

Fig. 3. Actual voltage signal $u$, the reconstructed signal $u_{r}$ and residual signal $u_{e}$
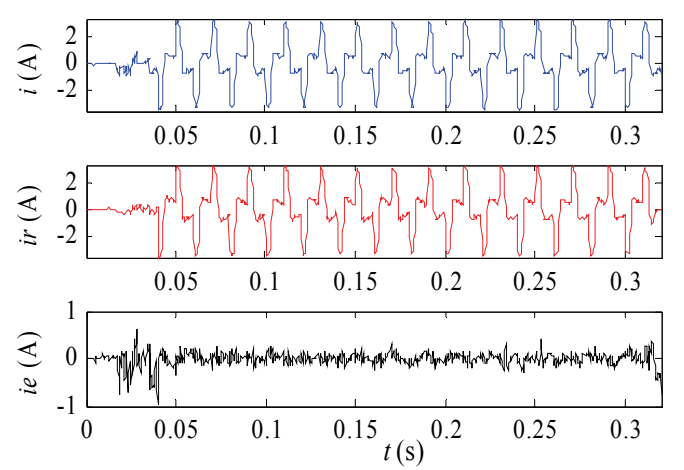

Fig. 4. Actual current signal $i$, the reconstructed signal $i_{r}$ and residual signal $i_{e}$

and current signals are carried out using the proposed method. The USB data acquisition card is used to acquire the voltage from the sensors, the Hall voltage sensor $(400 \mathrm{~V} / 5 \mathrm{~V})$ and Hall current sensor $(5 \mathrm{~A} / 5 \mathrm{~V})$ are used to collect the operating voltage and current of five personal computers respectively. The sampling frequency of 3200 $\mathrm{Hz}$ is also used here, and the signals to be analyzed have $N=1024$ samples ( 16 cycles, 0.32 s).

In Fig. 3, $u$ is the steady state voltage with harmonics acquired when computers are running, $u_{r}$ is the reconstructed voltage signal containing extracted components after 15 iterations by FMP method, and $u_{e}$ is the residual voltage that containing system noise and decomposing errors. In Fig. 4, $i$ is the initial unsteady state current with serious distortion, $i_{r}$ is the reconstructed current signal containing extracted components after 15 iterations, and $i_{e}$ is the residual current signal that containing system noise and decomposing errors. The parameters of the main components extracted from $u$ and $i$ are shown in Table 8 and Table 9 respectively.

It can be seen from the waveform of $u$ in Fig. 3 that the voltage distortion is not obvious, so we can preliminary deduce that the amplitude of harmonic or inter-harmonic is small. As it can be seen from Table 8, many harmonics are detected, but the main components are $3^{\text {rd }}$ and $9^{\text {th }}$ harmonics, and the amplitudes of the two harmonics are $3.4 \%$ and $1.6 \%$ of the fundamental component respectively. 
Table 8. Detection results of the actual voltage

\begin{tabular}{c|c|c|c|c|c}
\hline & $\begin{array}{c}\text { amplitude } \\
(\mathrm{V})\end{array}$ & $\begin{array}{c}\text { RMS } \\
(\mathrm{V})\end{array}$ & $\begin{array}{c}\text { frequency } \\
(\mathrm{Hz})\end{array}$ & $t_{\mathrm{s}}(\mathrm{s})$ & $t_{\mathrm{e}}(\mathrm{s})$ \\
\hline$u_{1}$ & 310.41 & 219.49 & 50.02 & 0.000 & 0.320 \\
\hline$u_{2}$ & 10.70 & 7.57 & 150.07 & 0.000 & 0.320 \\
\hline$u_{3}$ & 5.04 & 3.57 & 450.21 & 0.000 & 0.318 \\
\hline$u_{4}$ & 1.53 & 1.08 & 750.36 & 0.002 & 0.320 \\
\hline$u_{5}$ & 1.19 & 0.84 & 550.27 & 0.002 & 0.315 \\
\hline$u_{6}$ & 1.22 & 0.86 & 950.52 & 0.000 & 0.318 \\
\hline$u_{7}$ & 1.17 & 0.83 & 350.22 & 0.000 & 0.313 \\
\hline$u_{8}$ & 1.00 & 0.71 & 650.26 & 0.002 & 0.313 \\
\hline
\end{tabular}

Table 9. Detection results of the actual current

\begin{tabular}{c|c|c|c|c|c}
\hline & $\begin{array}{c}\text { amplitude } \\
(\mathrm{A})\end{array}$ & RMS $(\mathrm{A})$ & $\begin{array}{c}\text { frequency } \\
(\mathrm{Hz})\end{array}$ & $t_{\mathrm{s}}(\mathrm{s})$ & $t_{\mathrm{e}}(\mathrm{s})$ \\
\hline$i_{1}$ & 1.915 & 1.354 & 49.96 & 0.040 & 0.314 \\
\hline$i_{2}$ & 1.316 & 0.931 & 149.99 & 0.040 & 0.319 \\
\hline$i_{3}$ & 0.460 & 0.325 & 250.00 & 0.041 & 0.320 \\
\hline$i_{4}$ & 0.210 & 0.149 & 499.82 & 0.024 & 0.310 \\
\hline$i_{5}$ & 0.150 & 0.106 & 350.26 & 0.040 & 0.316 \\
\hline$i_{6}$ & 0.125 & 0.088 & 550.10 & 0.037 & 0.315 \\
\hline$i_{7}$ & 0.093 & 0.066 & 649.74 & 0.035 & 0.318 \\
\hline$i_{8}$ & 0.079 & 0.056 & 949.72 & 0.042 & 0.319 \\
\hline$i_{9}$ & 0.076 & 0.054 & 750.10 & 0.040 & 0.320 \\
\hline$i_{10}$ & 0.061 & 0.043 & 100.41 & 0.019 & 0.319 \\
\hline$i_{11}$ & 0.071 & 0.051 & 199.67 & 0.032 & 0.317 \\
\hline$i_{12}$ & 0.051 & 0.036 & 299.72 & 0.018 & 0.315 \\
\hline$i_{13}$ & 0.039 & 0.027 & 850.29 & 0.025 & 0.320 \\
\hline$i_{14}$ & 0.041 & 0.029 & 400.01 & 0.037 & 0.320 \\
\hline$i_{15}$ & 0.032 & 0.023 & 1249.98 & 0.043 & 0.312 \\
\hline
\end{tabular}

The amplitudes of the other components extracted from $u$ are all less then $0.5 \%$ of the fundamental component. Table 8 only shows the detection results of the components whose amplitudes are above $1 \mathrm{~V}$.

It is apparent from Fig. 4 that the distortion of current $i$ is serious, so we can deduce that the harmonic or interharmonic disturbances should accounted for a large proportion. As shown in Table 9, a large amount of harmonic currents are detected in $i$, of which $3^{\text {rd }}$ harmonic contributes the maximum proportion, the amplitude of $3^{\text {rd }}$ harmonic is $68.7 \%$ of the fundamental component, and the $5^{\text {th }}, 9^{\text {th }}, 7^{\text {th }}$ and $11^{\text {th }}$ harmonics are $24 \%, 11 \%, 7.8 \%$ and $6.5 \%$ of the fundamental component respectively. The smaller harmonics and inter-harmonics are not listed in this table. It can be seen that the content of harmonics are very high, which leads to serious current distortion. At the same time, the method detected the start and end time of the harmonic current, which is approximately coincide with the real time of the original current $i$.

From the analysis of the waveforms and data, it can be conclude that the proposed technique is effective in detecting steady and unsteady harmonics/inter-harmonics of field or practical power system signals.

\subsection{Execution time}

Often, the execution time of the algorithm is an important indicator. As an example, using Matlab software running on a PC with Intel Pentium Dual-core E6700 3.2 $\mathrm{GHz} \mathrm{CPU}$, the decomposition carried out 9 iterations for the signal in section 4.3 was performed within average time of $0.62 \mathrm{~s}$, and the average execution time of each iteration is about $0.069 \mathrm{~s}$. The decomposition carried out 15 iterations for the actual current signal in section 4.4 was performed within average time of $1.02 \mathrm{~s}$, and the average execution time of each iteration is about $0.068 \mathrm{~s}$. Therefore, the time cost of the proposed method is appropriate for computer based harmonic analyses.

\section{Conclusion}

In this paper, an attempt is made to detect the harmonic and inter-harmonic disturbances from power system signal using FMP method. The method presents fast detection of harmonics and inter-harmonics from electrical signals. Different simulation cases and the actual power system voltage and current signals are tested and analyzed, for all these cases, the method shown to be efficient, and presenting the following advantages: (1) It has clear physical significance. (2) It can detect the amplitude, frequency, phase and duration time of harmonic or interharmonic. (3) It can distinguish the inter-harmonic frequency adjacent to fundamental or harmonic frequency. (4) The detection performance almost does not affected by fundamental frequency variation. (5) It has better anti-noise performance. Finally we think the method provides an alternative way to harmonic and interharmonic analysis, and is suitable for the application of off-line fault signal analysis or power quality analysis etc.

\section{Acknowledgements}

This work was supported by the Natural Science Foundation of Hainan Province (No.20166227) and Science \& Technology Support Program of Qinhuangdao (No. 201302A042).

\section{References}

[1] Yilmaz, M. Ermis, and I. Cadirci, "Mediumfrequency induction melting furnace as a load on the power system," IEEE Transactions on Industry Applications, vol. 48, no. 4, pp. 1203-1214, Apr. 2012.

[2] Z. Leonowicz, T. Lobos, and J. Rezmer, "Advanced spectrum estimation methods for signal analysis in power electronics," IEEE Transactions on Industrial Electronics, vol. 50, no. 3, pp. 514-519, Mar. 2003.

[3] H. Wen, S. Guo, Z. Teng, F. Li, and Y. Yang, "Frequency estimation o f distorted and noisy signals 
in power systems by FFT-based approach," IEEE Transactions on Power System, vol. 29, no. 2, pp. 765-774, Feb. 2014.

[4] F. Jurado, and JR. Saenz, "Comparison between discrete STFT and wavelets for the analysis of power quality events," Electric Power System Research, vol. 62, no.3, pp. 192-199, Mar. 2002.

[5] R. I. Diego, and J. Barros, "Global method for timefrequency analysis of harmonic distortion in power systems using the wavelet packet transform," Electric Power Systems Research, vol. 79, no. 8, pp. 12261239, Aug. 2009.

[6] J. Macias and A. Gomez-Exposito, "Self-tuning of Kalman filters for harmonic computation," IEEE Transactions on Power Delivery, vol. 21, no. 1, pp. 501-503, Jan. 2006.

[7] C. Guo, Q.Z. Li, and J.M. He, "Segmentation Prony algorithm on harmonics and inter-harmonics detection of power networks," Power System Technology, vol. 34, no. 3, pp. 21-25, Mar. 2010.

[8] Y. H. Zhang, W. R. Duan and T. Y. Li, "Method to detect harmonics and inter-harmonics based on multiple-level EEMD and Wigner-Ville distribution," Advanced Technology of Electrical Engineering and Energy, vol. 34, no. 3, pp. 66-71, Mar. 2015.

[9] S. Jain and S. Singh, "Exact model order esprit technique for harmonics and inter-harmonics estimation," IEEE Transactions on Instrumentation and Measurement, vol. 61, no. 7, pp. 1915-1923, Jul. 2012.

[10] D. D. Ferreira, E. A. Nagata, S. C. Ferreira, J.M.D. Seixas and C.A. Duque, "Method based on independent component analysis for harmonic extraction from power system signals," Electric Power Systems Research, vol. 119, no. 2, pp. 19-24, Feb. 2015.

[11] L.L. Meng, C.D. Sun and X.D. Wang, "Harmonic/ inter-harmonic detection based on DNS-MUSIC function," Electric Power Automation Equipment, vol. 32, no. 2, pp. 89-94, Feb. 2012.

[12] Z.L. Xiao, R.X. Gong and S. Chen, "Detection of harmonics in power system based on modified Stransform," Power System Protection and Control, vol. 43, no. 3, pp. 89-94, Mar. 2015.

[13] G. Z. Fang, C. Yang and H. Zhao, "Detection of harmonic in power system based on FFT and wavelet packet," Power System Protection and Control, vol. 40, no. 5, pp. 75-79, May 2012.

[14] J. K. Chen, H. Y. Li, S. Y. Yang and B. Q. Kou, "Application of wavelet packet singularity entropy and PSD in power harmonics detection," Transactions of China Electro- technical Society, vol. 25, no. 8, pp. 193-199, Aug. 2010.

[15] C. Huang, Z.J. Zhu, Y.J. Cao and F. Wang, "A novel power system harmonic and interharmonic analysis method," Transactions of China Electrotechnical Society, vol. 28, no. 9, pp. 32-39, Sept. 2013.
[16] C.J. Huan, W.S. Cao, T.J. Chen, “Application of local mean decomposition in power quality disturbance detection," Electric Power Automation Equipment, vol. 33, no. 9, pp. 68-73, Aug. 2013.

[17] S. Y. Hou, W. Y. Zhang, T. Sun, "Analysis on matching features of power Quality disturbances based on orthogonal optimization of time-frequency atom decomposition," Power System Technology, vol. 37, no. 3, pp. 647-652, Mar. 2013.

[18] M. Li, G.X. Zhang and X.R. Wang, "Application of time-frequency atom method in inter-harmonics analysis," Power System Technology, vol. 33, no. 17, pp. 81-85, Aug. 2009.

[19] Y. J. Zhang, Q. W. Gong and X. Li, "Application of atomic decomposition based on PSO in interharmonics analysis," Power System Protection and Control, vol. 41, no.15, pp. 41-48, Aug. 2013.

[20] S. Mallat and Z. Zhang, "Matching pursuits with time-frequency dictionaries," IEEE Transactions on Signal Processing, vol. 41, no.12, pp. 3397-3415, Dec. 1993.

[21] J. A. Nelder, and R. Mead, "A simplex method for function minimization," Computer Journal, vol. 7, no.1, pp. 41-48, Apr. 1965.

[22] Z. Y. Cheng, J. K. Zuo, D. Liang and J. Q. Wang, "Detection of power system harmonic based on beamspace root-MUSIC," Transactions of Beijing Institute of Technology, vol. 34, no. 2, pp. 3397-3415, Feb. 2014.

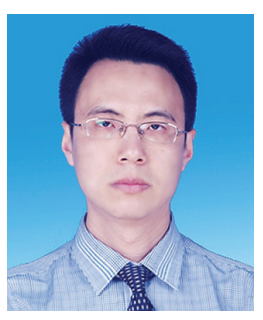

Lei Chen He received his M.S. degree in Information Engineering, YanShan University, Qinhuangdao, China, in 2007. Now he is an associate professor in Northeast Petroleum University, Qinhuangdao, China and is pursuing the Ph.D. degree in Electrical Engineering, YanShan University. His research interests include power quality analysis, intelligent detection and signal processing.

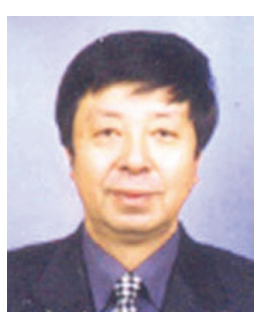

Dezhong Zheng He is a professor and doctoral supervisor in School of Electrical Engineering, Yanshan University, China. He is also the vice-president of the Artificial Intelligence Society in Hebei, China and the senior member of Chinese Institute of Electronics. His main research interests include intelligent information processing, intelligent control, virtual reality and pattern recognition. 


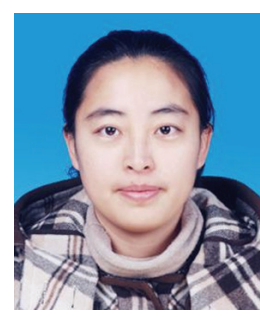

Shuang Chen She received her M.S. degree from Northwestern Polytechnical University, Xian, China, in 2006. She is now working as a faculty in Hebei Normal University of Science and Technology, Qinhuangdao, China. Her current research interests include intelligent optimization technology, information processing and embedded application system.

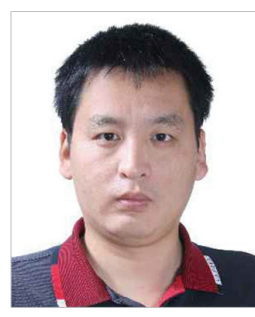

Baoru Han He received his M.S. degree in circuits and systems engineering from Yanshan University, China, in 2007 and Ph.D. degree in information and communication engineering from Hainan University, China, in 2016. He is currently an associate professor in Hainan Soft-ware Profession Institute, China. His research interests include digital watermarking, signal and image processing. 